\title{
Analysis of Healthcare Seeking Behavior
}

\author{
Chrissy Dangel ${ }^{\star 1}$, Steven Allgeier ${ }^{1}$, Adam Haas ${ }^{2}$ and Amanda Johnson ${ }^{2}$ \\ ${ }^{1}$ US EPA, Cincinnati, OH, USA; ${ }^{2} \mathrm{CSC}$ Science \& Engineering, Cincinnati, $\mathrm{OH}, \mathrm{USA}$
}

\section{Objective}

This paper describes analyses of health seeking behaviors from two surveillance datastreams: Poison Control Center (PCC) calls and Emergency Department (ED) visit records. These analyses were conducted in order to quantify behaviors following the development of symptoms after water contamination exposure and to understand the motivation, decision-making and timing behind healthcare seeking behaviors.

\section{Introduction}

Healthcare seeking behavior is important to understand when interpreting public health surveillance data, planning for healthcare utilization, or attempting to estimate or model consequences of an adverse event, such as widespread water contamination. Although there is evidence that factors such as perceived susceptibility and benefits affect healthcare seeking behavior, it is difficult to develop accurate assumptions due to a lack of published research on this topic. Current conceptual behavior models, such as the health belief model, are not easily translated into quantifiable terms.

\section{Methods}

For the PCC analysis, data was included from 480 calls from two PCCs from September 2012 to April 2013. Shortly following the calls, PCC callers were surveyed and results were merged with call data. A subset of callers surveyed was preferentially selected based on the reason for call or substance described in an attempt to gather more information on situations similar to a possible water contamination event. The primary outcome of interest was the method of obtaining the PCC phone number. Methods of obtaining the PCC phone number were categorized as research, professional referral, PCC sticker/ magnet, friend/family and other/non-specified.

The ED visit records analysis comprised over 71,000 visit records from five EDs from January 2001 to December 2011 in Cincinnati, $\mathrm{OH}$. In this analysis, the primary outcome of interest was means of arrival to the ED. ED arrival was dichotomized as Emergency Medical Services (EMS) utilization (yes/no). Healthcare seeking behaviors were categorized by characteristics such as age, disease category, insurance type, disposition as a severity indicator and time period.

For both data sets, exploratory data analysis was performed using contingency tables. Differences in the proportions of methods to obtain the PCC phone number and to means of ED arrival were compared across demographic variables and visit characteristics using chi-square tests of significance.

\section{Results}

The main finding from the PCC analysis was that insurance type was significantly associated with obtaining the PCC number $(p=0.002)$. Survey respondents with private or employer based insurance were 1.9 times as likely to obtain the number from a PCC sticker or magnet while those with government based insurance were 1.7 times as likely to obtain the number after a referral compared to other methods.

The ED study found that arrival to the ED by EMS was uncommon $(<30 \%)$ but varied by $\mathrm{ED}(p<0.001)$. EMS utilization was more common in the autumn season and peaked on Fridays and Saturdays. Age, disease category, insurance type and disposition were significantly associated with EMS utilization ( $p$ 's <0.001). Adults at least 64 years of age were 1.9 times as likely to utilize EMS compared to younger adults. Children ages $15-19$ were 2.8 times as likely to arrive by EMS compared to younger children. Patients arriving to the ED due to poisoning of drugs or toxic effects of nonmedical substances had the greatest EMS utilization. The effect of insurance type on EMS utilization varied by patient age. Adults with government based insurance and children with commercial based insurance were the most likely to utilize EMS. Disposition exhibited the strongest effect on EMS utilization. Children who were admitted were 7.7 times as likely to arrive by EMS. Adult patients were 5.7 times as likely to arrive by EMS if they were admitted. In a sensitivity analysis adjusting for disposition, the association between insurance type and EMS utilization was no longer significant for admitted patients $(p=0.105)$.

\section{Conclusions}

This study better defines the health seeking behaviors of patients experiencing symptoms analogous to those following water contamination and highlights the importance of using diverse outreach methods to promote PCC and EMS utilization.

\section{Keywords}

healthcare; behavior; poison control center; emergency department; analysis

\section{References}

1. Sheeren, P. \& Abraham, C The Health Belief Model, in predicting Health Behaviour (Conner, M. \& Norman, P. eds). Buckingham: Open University Press. 1995

\section{*Chrissy Dangel}

E-mail: dangel.chrissy@epa.gov 